FECHA DE

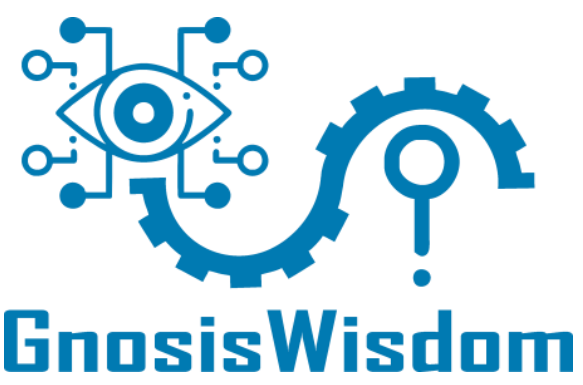

REVISTA DE INVESTIGACIÓN E INNOVACIÓN CIENTÍFICA Y TECNOLÓGICA

Artículo original

Volumen 1, Número 1, Enero-Abril 2021

\title{
LA COMPRENSIÓN DE LECTURA Y SU INFLUENCIA EN LA PRODUCCIÓN \\ LITERARIA DE LOS ESTUDIANTES DE LA FACULTAD DE CIENCIAS DE LA \\ EDUCACIÓN DE LA UNA - PUNO
}

\section{READING COMPREHENSION AND ITS INFLUENCE ON THE LITERARY PRODUCTION OF STUDENTS IN THE FACULTY OF EDUCATION SCIENCES OF THE UNA-PUNO}

\author{
Autor \\ Rosales Gallegos, Jorge ORCID \\ UNIVERSIDAD NACIONAL DEL ALTIPLANO
}

\section{Resumen}

El objetivo de este trabajo de investigación fue determinar la influencia de la comprensión de lectura en la producción literaria de los estudiantes de la Facultad de Ciencias de la Educación de la UNA - Puno. La muestra de estudio estuvo conformada por 44 estudiantes de VIII y IX semestre del Programa de Lengua, Literatura, Psicología y Filosofía. La metodología que se usó para alcanzar el objetivo general fue el modelo estadístico de regresión lineal simple y el coeficiente de correlación de Pearson. Para la recolección de los datos se aplicó la prueba escrita y la lista de cotejo. Los datos fueron tabulados y procesados en el Microsoft Excel y el software SPSS. Los resultados sugieren que cuando el estudiante incrementa en un punto su nivel de compresión de lectura, entonces su nivel de producción literaria sube en 0.8 puntos. Por lo tanto, se concluye que la comprensión de lectura influye significativamente en la producción literaria de los estudiantes de la Facultad de Ciencias de la Educación de la UNA - Puno, a un nivel de $99 \%$ de confianza.

Palabras clave: Comprensión de lectura, niveles de comprensión de textos, producción literaria, prosa y verso.

\begin{abstract}
he overall objective of this research work was to determine the influence of reading comprehension on the literary production of students of the Faculty of Educational Sciences of the UNA - Puno. The study sample consisted of 44 students of VIII and IX semester of the
\end{abstract}




\section{GnosisWisdam}

Program of Language, Literature, Psychology and Philosophy. The methodology used to achieve the general objective was the statistical model of simple linear regression and Pearson's correlation coefficient. The written test and the comparison list were used to collect the data. The data were tabulated and processed in Microsoft Excel and SPSS software. The results suggest that when the student increases his reading comprehension level by one point, then his literary production level rises by 0.8 points. Therefore, it is concluded that reading comprehension significantly influences the literary production of the students of the Faculty of Educational Sciences of the UNA - Puno, at a level of $99 \%$ confidence.

Keywords: Literary production, levels of comprehension of texts, reading comprehension, prose and verse.

\section{Introducción}

La comprensión de lectura y la producción de textos en la educación superior resultan de mucha importancia en la formación académica, ya que un estudiante necesita conocer estos temas para desenvolverse con solvencia académica a lo largo de su vida universitaria. En ese sentido, este trabajo de investigación se ajusta a nuestro contexto y a sus diversas necesidades, ya que en la actualidad resulta una necesidad el fomento de la comprensión y producción de textos en la educación superior. Asimismo, este estudio pretende motivar y concientizar a los docentes y estudiantes sobre la importancia de la comprensión y producción de textos en la formación universitaria.

La lectura de los textos involucra comprensión, interpretación e inferencia, lo que implica un proceso cognitivo muy complejo, que enfatiza el conocimiento de las estructuras linguísticas, la cultura y el contexto (Ugarriza, 2006). En esta línea, comprender un texto no es develar el significado de cada una de las palabras ni siquiera de las frases, o de la estructura general del texto; sino más bien generar una representación mental del referente del texto, es decir, producir un escenario o modelo mental de un mundo real $o$ hipotético en el cual el texto cobra sentido (Huamaní, 2008). Entonces, la comprensión de lectura es un proceso activo y constructivo de interpretación del significado del texto, donde se pone en juego una serie de operaciones y estrategias mentales para procesar la información que se recibe del texto, y luego se construye el significado del texto y se crea nueva información de la interacción entre la información obtenida del texto y el conocimiento previo del lector (Ramos, 2010). En ese sentido, la comprensión de lectura se lleva a cabo por las inferencias que el lector es capaz de realizar formando y comprobando hipótesis acerca de lo que se trata el texto, mediante esquemas mentales que permitan relacionar el mensaje del texto 


\section{REVISTA DE INVESTIGACIÓN E INNOVACIÓN}

CIENTÍFICA Y TECNOLÓGICA

Volumen 2, Número 1, Enero-Abril 2021

Fecha de publicación: 15-03-2021 - Fecha de aceptación:14-02-2021

\section{GnasisWisdam}

con los conocimientos previos (Caicedo, 2007).

Asimismo, las inferencias permiten establecer conexiones entre el muevo material que exhibe el texto y el conocimiento previo, ya existente en la memoria (Zuazo, 2017). El conocimiento previo está constituido no solamente por lo que el sujeto sabe sobre el tema específico, sino también por la forma en que está organizado su conocimiento, los instrumentos de asimilación de que dispone por su competencia lingüística en general y el conocimiento de la lengua en particular (De Zunino, 1985). En ese sentido, para leer necesitamos, simultáneamente, manejar con soltura las habilidades de descodificación y aportar al texto nuestros objetivos, ideas y experiencias previas (Solé, 1994); ya que la comprensión lectora es el proceso de elaborar el significado por la vía de aprender las ideas relevantes del texto y relacionarlas con las ideas que ya se tienen (Cooper, 1998). En consecuencia, entender un texto significa incorporar elementos a los que ya se tienen y hacer una interpretación razonable (Giovannini, 1996) e interpretar el mensaje más allá de la palabra impresa (Bell, 1997).

Por otro lado, la producción está referida y asociada con las ideas de originar, ocasionar y fabricar (Pérez \& Merino, 2008). En ese sentido, la producción literaria e intelectual se refiere a la creación y génesis de una obra que pueda reflejar los acontecimientos políticos, económicos, sociales, culturales y religiosos (Amaro \& Cabrera, 2005). Este autor agrega que un texto literario siempre incluirá ciertas marcas que revelen su contexto; de modo que es importante que el lector pueda descifrar estas marcas para poder llegar a una lectura más profunda. Por otro lado, los textos son leídos e interpretados dependiendo de la disposición anímica, la edad, las áreas de interés, las experiencias de vida y las lecturas anteriores (Real, et al, 2004). En consecuencia, la producción literaria de parte de los estudiantes de pregrado ayuda a asumir posturas y formular diversas opiniones de acuerdo a las diferentes temáticas de interés que se presentan en la sociedad (Schmitt, 1998).

Ramos (2010), en su trabajo de investigación, propone a las universidades e institutos pedagógicos incorporar a sus currículos un enfoque más próximo al desarrollo del niño; concretamente, al periodo de los primeros siete años, cruciales para su formación como lector y redactor. Asimismo, invoca a declinar las prácticas tradicionales, que condicionan el fracaso de lectores activos y reflexivos desde la educación inicial y que explican, en parte, por qué los universitarios y profesionales peruanos leen poco o se limitan a escribir textos breves. En esa línea, expone las dificultades que tiene el alumno para emplear la redacción como una forma personal de procesar información y como una herramienta para interactuar con su entorno. Así mismo, Escalante (2008) pág. 45 
Volumen 2, Número 1, Enero-Abril 2021 Fecha de publicación: 15-03-2021 - Fecha de aceptación:14-02-2021

\section{GnasisWisdam}

plantea la idea de que la Comprensión Lectora mejora significativamente el Rendimiento Académico de los estudiantes, por ello es necesario que los docentes realicen capitaciones, lecturas de bibliografía relacionada con la comprensión lectora y su enseñanza. Por otro lado, Huancas (2019) comprueba que existe una relación significativa entre la creatividad y la producción literaria por parte de los estudiantes de dicha institución.

En Argentina, Hauy (2014) concluye que la lectura literaria, aparte de desarrollar un enfoque subjetivo en los estudiantes, también aporta en la formación ética, lo cual fomenta una actitud crítica ante los aspectos de la cultura y la sociedad. Asimismo, en Colombia, Rueda y Turriago (2003) llegan a la conclusión de que enfocarse en nuevas propuestas pedagógicas a través del manejo de la literatura conlleva a mejorar el desarrollo del pensamiento, así como de las habilidades en la competencia gramatical, y fomenta el desarrollo de hábitos en la lectura y escritura; demostrando así la efectividad de la producción literaria como proceso para mejorar el pensamiento de los estudiantes. Por otro lado, en Perú, Bedoya (2019) concluye que un buen nivel de comprensión de lectura influye significativamente en el pensamiento crítico, sobre aspectos relacionados con la sociedad actual.

Así mismo, Núñez (2016) concluye que existe una relación positiva y significativa entre el dominio semántico y la comprensión lectora en los estudiantes del segundo ciclo de la especialidad de Lengua y Literatura, en la Facultad de Educación de la Universidad Nacional Federico Villarreal, Lima. Finalmente, Narváez (2016) demuestra que con la aplicación del método de análisis transversal se logró significativamente la comprensión integral de textos narrativos literarios en los estudiantes del $3^{\circ}$ año de la mención de Lengua y Literatura de la Escuela Académico-Profesional de Educación Secundaria de la Facultad de Educación y Ciencias de la Comunicación.

\section{Materiales y Métodos}

Población y muestra de estudio

La población estuvo constituida por los estudiantes de la especialidad de Lengua, Literatura, Psicología y Filosofía de la Escuela Profesional de Educación Secundaria, de la Facultad de Ciencias de la Educación, específicamente fueron los estudiantes del octavo y noveno semestre.

Tabla 1. Población de estudio

\begin{tabular}{lccc} 
& \multicolumn{2}{c}{ Estudiantes } & Total \\
\cline { 2 - 4 } & Varones & Mujeres \\
\hline VIII & 11 & 10 & 21 \\
semestre & & & \\
XI semestre & 12 & 11 & 23 \\
Total & 23 & 21 & 44 \\
\hline
\end{tabular}

Fuente: Registro de matrícula 2019

Dado que el tamaño de la población es reducido, no se trabajó con ninguna muestra. Descripción de los objetivos

Para alcanzar el primer objetivo específico se usó el método descriptivo. Los datos 
Volumen 2, Número 1, Enero-Abril 2021 Fecha de publicación: 15-03-2021 - Fecha de aceptación:14-02-2021

\section{GnasisWisdam}

fueron recogidos con el instrumento de la prueba escrita de comprensión de textos que corresponde a la técnica de la evaluación. Una vez recopilados los datos, se tabularon mediante tablas de frecuencias de la estadística descriptiva.

Para alcanzar el segundo objetivo específico se utilizó el método descriptivo a través de tablas de frecuencias. Los datos fueron recogidos con el instrumento de la guía de observación, que corresponde a la técnica de la observación. Después de recopilar los datos, se procedió con la tabulación y elaboración de tablas de frecuencias.

Para alcanzar el objetivo general se utilizó la técnica estadística de la regresión lineal. Con esta técnica se determinó el grado de influencia que ejerce la comprensión de lectura en la producción literaria de los estudiantes de Lengua, Literatura, Psicología y Filosofía de la Universidad Nacional del Altiplano. La regresión lineal permitió cuantificar el cambio en el nivel de producción literaria conforme cambia el nivel de comprensión de lectura. Este modelo presenta la siguiente notación:

$$
\mathrm{Y}=\mathrm{a}+\mathrm{bX}+\mathrm{e}
$$

Donde:

Y : Variable dependiente

a : Es el valor de la ordenada donde la

línea de regresión se intercepta con el eje de ordenada

b : Es el coeficiente de regresión (pendiente de la línea recta)

X : Variable independiente

$\underline{\mathrm{e}}$

: Es el error
Previo al cálculo de la regresión, se estimó el coeficiente de correlación de Pearson, para ver el grado de relación entre la comprensión de lectura y la producción literaria, mediante la siguiente fórmula:

$$
r=\frac{n \sum x y-\left(\sum x\right)\left(\sum y\right)}{\sqrt{\left.n \sum x^{2}-\left(\sum x\right)^{2}\right]\left[n \sum y^{2}-\left(\sum y\right)^{2}\right]}}
$$

\section{Resultados y Discusión}

Nivel de comprensión de lectura de los estudiantes de la Facultad de Ciencias de la Educación (UNA-Puno)

En el nivel literal, la mayoría (57\%) de los estudiantes de la Facultad de Ciencias de la Educación (UNA-Puno), en la comprensión de textos en prosa, se ubica en la escala Logro destacado (con notas entre 18 y 20 puntos). En el nivel inferencial, el $40 \%$ de estudiantes se ubica en la escala En proceso (con notas entre 11 y 13 puntos). En el nivel crítico, el $47 \%$ de estudiantes se ubica en la escala En proceso, con notas entre 11 y 13 puntos (Tabla 2). Dichos resultados indican que los estudiantes comprenden mejor literalmente un texto, pero tienen ciertas deficiencias en la comprensión inferencial y crítico.

Tabla 2. Nivel de comprensión de lectura de los estudiantes de la Facultad de Ciencias de la Educación (UNA-Puno) en prosa

\begin{tabular}{lllllll}
\hline & Intervalo & & $\begin{array}{l}\text { Nivel } \\
\text { literal }\end{array}$ & $\begin{array}{l}\text { Nivel } \\
\text { inferencial }\end{array}$ & $\begin{array}{l}\text { Nivel } \\
\text { crítico }\end{array}$ & Promedio \\
\hline $\begin{array}{l}\text { Logro } \\
\text { destacado }\end{array}$ & {$[20-18]$} & f & 17 & 5 & 6 & 9 \\
Logro & & $\%$ & $57 \%$ & $17 \%$ & $20 \%$ & $31 \%$ \\
previsto & {$[17-14]$} & f & 7 & 10 & 1 & 6 \\
& & $\%$ & $23 \%$ & $33 \%$ & $3 \%$ & $20 \%$ \\
& {$[13-11]$} & f & 5 & 12 & 14 & 10 \\
\hline
\end{tabular}


Volumen 2, Número 1, Enero-Abril 2021 Fecha de publicación: 15-03-2021 - Fecha de aceptación:14-02-2021

\section{GnasisWisdam}

$$
\begin{aligned}
& \begin{array}{llllll}
\text { proceso } & \% & 17 \% & 40 \% & 47 \% & 34 \%
\end{array} \\
& \begin{array}{lllllll}
\text { En inicio } & {[10-0]} & \mathrm{f} & 1 & 3 & 9 & 4 \\
& & \% & 3 \% & 10 \% & 30 \% & 14 \%
\end{array} \\
& \begin{array}{llllll}
\text { Total } & \mathrm{f} & 30 & 30 & 30 & 30 \\
& & 100 & & &
\end{array}
\end{aligned}
$$

Fuente: Resultados de la prueba escrita.

Según los resultados, la tendencia a producir textos en este estilo, especialmente en cuento, será realizado de una manera eficiente y factible. En la misma línea, Bedoya (2019) afirma que existe una relación significativa entre la comprensión lectora y la disposición hacia el pensamiento crítico en los estudiantes de la Facultad de Educación de una universidad pública del Norte del Perú. Los estudiantes demuestran que despliegan procesos cognoscitivos, eficaces, organizados y funcionales que le permiten entender y organizar los propios pensamientos relacionados con la comprensión lectora. En efecto, los estudiantes durante la comprensión lectora activan procesos cognitivos que están vinculados con las disposiciones del pensamiento crítico. En base a estos estudios podemos afirmar que una buena comprensión de textos en prosa influirá en forma significativa en la producción literaria. Por su parte, Vallés (2005) señala que si la lectura en sí misma resulta un acto placentero, el lector experimentará un estado de ánimo positivo, favorable y que, en los casos de mayor implicación y disfrute ha venido a definirse como un estado de mental y emocional con sensación de gran bienestar y satisfacción que permite una mejor realización de las tareas habituales.
Finalmente, Zorrilla (2005) agrega que la comprensión lectora es considerada como el conjunto de las fases que intervienen en los procesos implicados en la formación, elaboración, notificación e integración de dichas estructuras de conocimiento

En cuanto a la comprensión de textos en verso, en el nivel literal, la mayoría (63\%) de estudiantes se ubica en la escala Logro destacado (con notas entre 18 y 20 puntos). En el nivel inferencial, el $43 \%$ de estudiantes se ubica en la escala Logro previsto (con notas entre 14 y 17 puntos). En el nivel crítico, el $57 \%$ de estudiantes se ubica en la escala En proceso, con notas entre 11 y 13 puntos (Tabla 3). Estos resultados indican que los estudiantes están más preparados para comprender literalmente los textos en verso, que inferencial y críticamente.

Tabla 3. Nivel de comprensión de lectura de

\begin{tabular}{|c|c|c|c|c|c|c|}
\hline Escalas & Intervalo & & $\begin{array}{l}\text { Nivel } \\
\text { literal }\end{array}$ & $\begin{array}{l}\text { Nivel } \\
\text { inferencial }\end{array}$ & $\begin{array}{l}\text { Nivel } \\
\text { crítico }\end{array}$ & Promedio \\
\hline \multirow{2}{*}{$\begin{array}{l}\text { Logro } \\
\text { destacado }\end{array}$} & {$[20-18]$} & $\mathrm{f}$ & 19 & 5 & 9 & 11 \\
\hline & & $\%$ & $63 \%$ & $17 \%$ & $30 \%$ & $37 \%$ \\
\hline \multirow{2}{*}{$\begin{array}{l}\text { Logro } \\
\text { previsto }\end{array}$} & {$[17-14\rceil$} & $\mathrm{f}$ & 9 & 13 & 0 & 7 \\
\hline & & $\%$ & $30 \%$ & $43 \%$ & $0 \%$ & $24 \%$ \\
\hline \multirow{2}{*}{$\begin{array}{l}\text { En } \\
\text { proceso }\end{array}$} & {$[13-11]$} & $\mathrm{f}$ & 2 & 12 & 17 & 10 \\
\hline & & $\%$ & $7 \%$ & $40 \%$ & $57 \%$ & $34 \%$ \\
\hline \multirow[t]{2}{*}{ En inicio } & {$[10-0]$} & $\mathrm{f}$ & 0 & 0 & 4 & 1 \\
\hline & & $\%$ & $0 \%$ & $0 \%$ & $13 \%$ & $4 \%$ \\
\hline \multirow{2}{*}{ Total } & & $\mathrm{f}$ & 30 & 30 & 30 & 30.0 \\
\hline & & $\%$ & $100 \%$ & $100 \%$ & $100 \%$ & $100 \%$ \\
\hline
\end{tabular}
los estudiantes de la Facultad de Ciencias de la Educación (UNA-Puno) en verso

Fuente: Elaboración propia sobre la base de los resultados de la prueba escrita. 
Volumen 2, Número 1, Enero-Abril 2021 Fecha de publicación: 15-03-2021 - Fecha de aceptación:14-02-2021

\section{GnasisWisdam}

Respecto a la comprensión de textos en verso, afirmamos que el mayor porcentaje se encuentra en un logro destacado y en un logro en proceso, lo cual nos indica que la tendencia a producir textos de este tipo resultará significativa. Núñez (2016) afirma que existe una relación positiva $\mathrm{y}$ significativa entre el dominio semántico y la comprensión lectora en los estudiantes del segundo ciclo de la especialidad de Lengua y Literatura, Facultad de Educación de la Universidad Nacional Federico Villarreal, Lima. Esto significa que la comprensión lectora que presentan los alumnos mayoritariamente en un nivel medio, está en directa relación con el dominio semántico que presentan medianamente en lo referido a vocabulario en contexto, sinónimos en contexto, antónimo, parónimo, homónimos, hiperónimos e hipónimos y marcadores textuales. En base a este estudio, podemos afirmar que los estudiantes comprenden el lenguaje que se presentan en los textos escritos en verso. Estos datos coinciden con lo hallado por Echavarría y Gastón (2000), quienes demuestran que los estudiantes presentan dificultades en la comprensión de los textos a nivel de la selección y jerarquización de la información relevante (macroestructura) y en la captación de la intencionalidad comunicativa del autor, que se refleja en la estructura del texto (superestructura). Al respecto, Ochoa y Aragón (2004) encontraron una relación significativa y positiva entre el funcionamiento metacognitivo de estudiantes universitarios y los niveles de comprensión de artículos científicos tipo ensayo teórico y reporte de investigación, de tal modo que a mayor nivel metacognitivo, mayor nivel de comprensión lectora, y viceversa

En cuanto al nivel de comprensión de lectura de los estudiantes de la Facultad de Ciencias de la Educación (UNA-Puno), tanto en prosa como en verso, el $67 \%$ de estudiantes se ubica en la escala Logro previsto, con notas entre 14 y 17 puntos. El 3\% de estudiantes se ubica en la escala En inicio, con notas entre 0 y 10 puntos. El 23\% de estudiantes se ubica en la escala En proceso, con notas entre $11 \mathrm{y}$ 13 puntos. El $7 \%$ de estudiantes se ubica en la escala Logro destacado, con notas entre 18 y 20 puntos (Tabla 4).

Tabla 4. Nivel de comprensión de lectura de los estudiantes de la Facultad de Ciencias de la Educación (UNA-Puno)

\begin{tabular}{lcll}
\hline Escalas & Intervalo & Frecuencia & Porcentaje \\
\hline $\begin{array}{l}\text { Logro } \\
\text { destacado }\end{array}$ & {$[20-18]$} & 2 & $7 \%$ \\
$\begin{array}{l}\text { Logro } \\
\text { previsto }\end{array}$ & {$[17-14]$} & 20 & $67 \%$ \\
En proceso & {$[13-11]$} & 7 & $23 \%$ \\
En inicio & {$[10-0]$} & 1 & $3 \%$ \\
Total & & 30 & $100 \%$ \\
\hline
\end{tabular}

Fuente: Resultados de la prueba escrita.

Respecto a la comprensión global en los tres niveles, afirmamos que los estudiantes de la Facultad de Ciencias de la Educación, se ubica en la escala de logro previsto, lo cual nos indica que la mayoría de estudiantes están en un nivel medio de comprensión, por lo cual la influencia a la producción literaria, 
Volumen 2, Número 1, Enero-Abril 2021 Fecha de publicación: 15-03-2021 - Fecha de aceptación:14-02-2021

\section{GnasisWisdam}

será de una manera significativa. Así también, Nina (2018) sostiene que la comprensión lectora influye significativamente en el rendimiento académico. A lo cual en las recomendaciones que se hacen en la investigación se propone que se debe de implementar acciones y medidas que impulsen a desarrollar la comprensión de lectura en el nivel superior, con la finalidad de que este aspecto influirá de manera positiva y significativa en muchos aspectos que se vinculan a la formación académica y profesional. Por su parte, Flores (2011), señala una lectura frecuente de textos literarios, con un propósito recreativo, se asocia a niveles altos de competencia en los ámbitos matemático, lingüístico y científico. En cambio, la frecuencia con que se practica una lectura instrumental, dirigida a la mera localización de información o a la participación en situaciones de comunicación a través de Internet, no se vincula a diferentes niveles de aprendizaje. El nivel de producción literaria de los estudiantes de la Facultad de Ciencias de la Educación (UNA-Puno)

En cuanto al nivel de producción literaria de los estudiantes de la Facultad de Ciencias de la Educación (UNA-Puno) en verso, el 53\% de estudiantes se ubica en la escala Logro previsto, con notas entre 14 y 17 puntos. El $7 \%$ de estudiantes se ubica en la escala Logro destacado, con notas entre 18 y 20 puntos. El 27\% de estudiantes se ubica en la escala En proceso, con notas entre 11 y 13 puntos. El $13 \%$ de estudiantes se ubica en la escala En inicio, con notas entre 0 y 10 puntos (Tabla 5).

Tabla 5. Nivel de producción literaria de los estudiantes de la Facultad de Ciencias de la Educación (UNA-Puno) en verso

\begin{tabular}{llll}
\hline Escalas & Intervalo & Frecuencia & Porcentaje \\
\hline Logro destacado & {$[20-18]$} & 2 & $7 \%$ \\
Logro previsto & {$[17-14]$} & 16 & $53 \%$ \\
En proceso & {$[13-11]$} & 8 & $27 \%$ \\
En inicio & {$[10-0]$} & 4 & $13 \%$ \\
Total & & 30 & $100 \%$ \\
\hline
\end{tabular}

Fuente: Elaboración propia sobre la base de los resultados de la guía de observación La producción literaria en verso nos indica que la mayoría de estudiantes se ubican en un logro previsto, con lo cual se afirma que se cumple con los indicadores establecidos en la operacionalización de variables; así mismo, Huancas (2019) afirma que existe una relación significativa entre la creatividad y la producción literaria poética, a nivel de la prueba total y por las dimensiones: planificación, textualización y revisión en la producción de textos en verso. Ante lo cual se evidencia que el nivel de comprensión de lectura que tienen los estudiantes de la Facultad de Ciencias de la Educación favorece de manera significativa en la producción literaria en los textos escritos en verso. Lo cual cobra importancia porque la literatura se constituye en uno de los canales de participación cultural del ciudadano, lo mismo en su calidad de receptor que de eventual creador, y trasciende mucho más que otro tipo de interacciones comunicativas, el enriquecimiento de los 


\section{REVISTA DE INVESTIGACIÓN E INNOVACIÓN}

CIENTÍFICA Y TECNOLÓGICA

Volumen 2, Número 1, Enero-Abril 2021

Fecha de publicación: 15-03-2021 - Fecha de aceptación:14-02-2021

\section{GnasisWisdam}

usos verbales hasta afectar al mundo de los valores personales y de las opciones ideológicas (Rodríguez, 2014).

En cuanto al nivel de producción literaria de los estudiantes de la Facultad de Ciencias de la Educación (UNA-Puno) en prosa, el 67\% de estudiantes se ubica en la escala Logro previsto, con notas entre 14 y 17 puntos. El $3 \%$ de estudiantes se ubica en la escala En inicio, con notas entre 0 y 10 puntos. El $20 \%$ de estudiantes se ubica en la escala En proceso, con notas entre 11 y 13 puntos. El $10 \%$ de estudiantes se ubica en la escala Logro destacado, con notas entre 18 y 20 puntos (Tabla 6).

Tabla 6. Nivel de producción literaria de los estudiantes de la Facultad de Ciencias de la Educación (UNA-Puno) en prosa

\begin{tabular}{llll}
\hline Escalas & Intervalo & Frecuencia & Porcentaje \\
\hline $\begin{array}{l}\text { Logro } \\
\text { destacado }\end{array}$ & {$[20-18]$} & 3 & $10 \%$ \\
Logro & & & \\
previsto & {$[17-14]$} & 20 & $67 \%$ \\
En proceso & {$[13-11]$} & 6 & $20 \%$ \\
En inicio & {$[10-0]$} & 1 & $3 \%$ \\
Total & & 30 & $100 \%$
\end{tabular}

Fuente: Elaboración propia sobre la base de los resultados de la guía de observación

La producción literaria de los textos escritos en prosa, indica que el mayor porcentaje se ubica en un logro previsto, en un $67 \%$, lo cual nos indica que los estudiantes si cumplen con los indicadores propuestos en la operacionalización de variables, específicamente, en la producción literaria (textos en prosa). Por su parte, Rueda y Turriago (2003) afirman que la producción literaria en estudiantes de educación superior, tiene un nivel muy diferenciado, de acuerdo a las necesidades que presentan, según el origen. La producción literaria, sobre todo en textos narrativos, tienden a fomentar el aspecto crítico. En este sentido, la acción escolar es inconcebible sin la escritura, porque el quehacer académico, a través de materiales escritos, no sólo se extrae y difunde el conocimiento, sino que además se demuestra la posesión del mismo mediante actividades lingüísticas que requieren de la escritura, tales como: ensayos, minutas de aprendizaje, resúmenes, monografías, entre otros (Caldera \& Bermúdez, 2007).

En cuanto al nivel de producción literaria de los estudiantes de la Facultad de Ciencias de la Educación (UNA-Puno), el $57 \%$ de estudiantes se ubica en la escala Logro previsto, con notas entre 14 y 17 puntos. El $0 \%$ de estudiantes se ubica en la escala En inicio, con notas entre 0 y 10 puntos. El $37 \%$ de estudiantes se ubica en la escala En proceso, con notas entre 11 y 13 puntos. El $7 \%$ de estudiantes se ubica en la escala Logro destacado, con notas entre 18 y 20 puntos (Tabla 7).

Tabla 7. Nivel de producción literaria de los estudiantes de la Facultad de Ciencias de la Educación (UNA-Puno)

\begin{tabular}{lccc}
\hline Escalas & Intervalo & Frecuencia & Porcentaje \\
\hline $\begin{array}{l}\text { Logro } \\
\text { destacado }\end{array}$ & {$[20-18]$} & 2 & $7 \%$ \\
$\begin{array}{l}\text { Logro } \\
\text { previsto }\end{array}$ & {$[17-14]$} & 17 & $57 \%$ \\
En proceso & {$[13-11]$} & 11 & $37 \%$ \\
& & &
\end{tabular}


Volumen 2, Número 1, Enero-Abril 2021

Fecha de publicación: 15-03-2021 - Fecha de aceptación:14-02-2021

\section{GnasisWisdam}

En inicio

$[10-0] \quad 0$

$0 \%$

Total

30

$100 \%$

Fuente: Elaboración propia sobre la base de los resultados de la guía de observación

Respecto a la producción literaria en general por parte de los estudiantes de la Facultad de Ciencias de la Educación de la UNA - Puno, se afirma que se encuentran en un logro previsto, por lo cual indicamos que la influencia de la comprensión de lectura es significativa en la producción literaria (cuentos y poemas). Hauy (2014) afirma que leer textos literarios conlleva a mejorar en la didáctica de producción textual, ante lo cual se precisa que se debe de fortalecer estas necesidades, para lograr que la producción textual en estudiantes de educación superior sea constructiva en los aspectos de análisis y crítica. Por otra parte, Ramos (2011) también considera estas necesidades, en las cuales hace hincapié por la mejora de aspectos que colaboren en la producción textual de textos literarios.

Correlación y regresión

Los resultados del gráfico de dispersión indican que existe relación positiva entre ambas variables, ya que los puntos se concentran en torno a la línea de tendencia (Figura 1). Del mismo modo, el P-valor (0.000) del coeficiente de correlación de Pearson resultó menor que el valor de nivel de significancia (0.01), por lo tanto, existe relación positiva alta en términos estadísticos entre la comprensión de lectura y la producción literaria, a un nivel de $99 \%$ de confianza (Tabla 8).

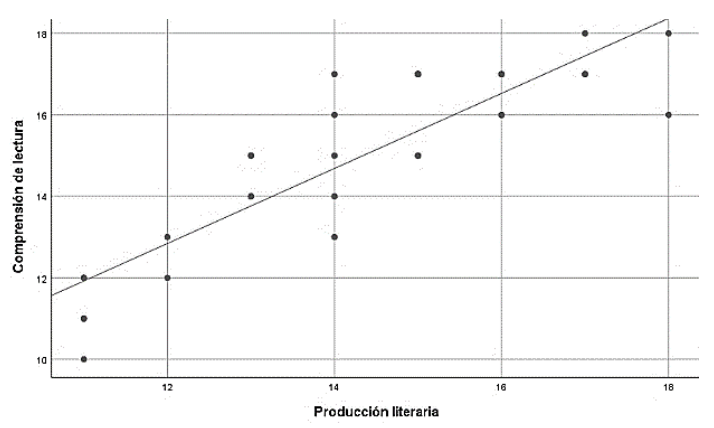

Figura 1. Diagrama de dispersión entre comprensión de lectura y producción literaria

Tabla 8. Correlación de Pearson entre comprensión de lectura y producción literaria

\begin{tabular}{cccc}
\hline & & $\begin{array}{c}\text { Comprensión } \\
\text { de lectura }\end{array}$ & Producción literaria \\
\hline $\begin{array}{c}\text { Comprensión } \\
\text { de lectura }\end{array}$ & $\begin{array}{c}\text { Correlación } \\
\text { de Pearson } \\
\text { Sig. } \\
\text { (bilateral) } \\
\text { N }\end{array}$ & 1 & 0.879 \\
& 30 & 0.000 \\
Producción & $\begin{array}{c}\text { Correlación } \\
\text { de Pearson } \\
\text { Siteraria }\end{array}$ & 0.879 & 30 \\
& $\begin{array}{c}\text { Silateral) } \\
\mathrm{N}\end{array}$ & 0.000 & 1 \\
& 30 & 30 \\
\hline
\end{tabular}

Fuente: Datos estimados con SPSS

A nivel global, el modelo de regresión lineal aplicado es significativo en términos estadísticos, a un nivel de 99\% de confianza, ya que el $\mathrm{P}$-valor de la prueba $\mathrm{F}$ resultó 0.000 . Este valor es menor que 0.01 . Por otro lado, a nivel individual, el valor estimado del coeficiente de la variable comprensión de lectura es significativo en términos estadísticos, a un nivel de 99\% de confianza, 
Volumen 2, Número 1, Enero-Abril 2021 Fecha de publicación: 15-03-2021 - Fecha de aceptación:14-02-2021

\section{GnosisWisdam}

ya que el P-valor de la prueba t (0.000) resultó menor que 0.01 . Así mismo, el $\mathrm{R}^{2}(\mathrm{R}$ cuadrado) estimado fue 0.773. Lo cual significa que el $77 \%$ de variación del nivel de producción literaria es explicada por la comprensión de lectura. Finalmente, el coeficiente de la variable comprensión de lectura resultó 0.839 . Lo cual significa que si el estudiante incrementa en un punto su nivel de compresión de lectura, entonces su nivel de producción literaria subirá en 0.8 puntos (Tabla 9).

Tabla 9. Regresión lineal simple

\begin{tabular}{llll}
\hline & Coeficiente & Prueba t & P-valor \\
\hline $\begin{array}{l}\text { Comprensión } \\
\text { de lectura }\end{array}$ & 0.839 & 9.751 & 0.000 \\
& & & \\
& Prueba F & 95.088 & \\
& $\begin{array}{l}\text { P-valor } \\
\text { R }\end{array}$ & 0.000 & \\
& cuadrado & 0.773 & \\
\hline
\end{tabular}

Fuente: Resultados obtenidos con el software SPSS.

Los resultados de esta investigación son corroborados por Zuazo (2017), quien concluyó que las estrategias de la comprensión lectora utilizadas por el docente de la Institución Educativa María Victoria Rumiche Fiestas N¹4083 (Sechura) influyen en la producción de textos narrativos de los estudiantes. Lo cual significa que los alumnos tienen la capacidad para comprender y a la vez producir textos teniendo en cuenta la coherencia textual y el uso correcto de la ortografía. Al respecto, Caldera y Bermúdez (2007) señalan que la lectura y la escritura son procesos interdependientes porque se escribe para que alguien lea y sin la lectura el proceso de escribir queda incompleto, por lo tanto, no es posible una escritura eficiente sin una lectura profunda que la anteceda.

\section{Conclusiones}

Según los resultados obtenidos, la comprensión de lectura influye significativamente en la producción literaria de los estudiantes de la Facultad de Ciencias de la Educación de la UNA - Puno, a un nivel de $99 \%$ de confianza. Esta afirmación se sustenta en el modelo estadístico de la regresión lineal que se llevó a cabo para esta investigación, donde se determinó que cuando el estudiante incrementa en un punto su nivel de compresión de lectura, entonces su nivel de producción literaria sube en 0.8 puntos, es decir, a mayor nivel de comprensión de lectura, el estudiante mejora el nivel de producción literaria.

En cuanto al nivel de comprensión de lectura, más de la mitad de estudiantes (67\%) de la Facultad de Ciencias de la Educación (UNA-Puno) se ubica en la escala Logro previsto, es decir, con notas entre 14 y 17 puntos; por otro lado, un porcentaje mínimo (3\%) de estudiantes se ubica en la escala En inicio, es decir, con notas entre $0 \mathrm{y}$ 10 puntos. Asimismo, se ha determinado que los estudiantes son más competentes en el nivel literal que en los niveles inferencial y crítico.

En cuanto al nivel de producción literaria, más de la mitad de estudiantes $(57 \%)$ de la Facultad de Ciencias de la Educación (UNA- 
Volumen 2, Número 1, Enero-Abril 2021 Fecha de publicación: 15-03-2021 - Fecha de aceptación:14-02-2021

\section{GnasisWisdam}

Puno) se ubica en la escala Logro previsto, es decir con notas entre 14 y 17 puntos. Por otra parte, no hay ningún estudiante que se ubique en la escala En inicio (con notas entre 0 y 10 puntos). Estos resultados sugieren que los estudiantes son competentes tanto en la producción de cuentos como en poemas.

\section{Conflicto De Intereses}

El autor no tiene conflicto de intereses.

\section{Agradecimientos}

A mi familia, a mis padres, a mis docentes y compañeros; gracias a ellos no hubiera sido posible este trabajo de investigación.

\section{Referencia}

Amaro, L., \& Cabrera, M. (2005). Lengua Castellana y Comunicación 2 (Santillana, Ed.). Santiago de Chile.

Bedoya, B. (2019). Relación entre la comprensión lectora y la disposición hacia el pensamiento crítico en los estudiantes de la Facultad de Educación de una universidad pública del norte del Perú. Universidad Peruana Cayetano Heredia.

Caicedo, L. (2007). Aplicación de estrategias meta cognitivas para incrementar la comprensión lectora en alumnos del 4to básico.

Caldera, R., \& Bermúdez, A. (2007). Alfabetización académica: comprensión y producción de textos. Educere, 11(37), 247-255.
Cooper, J.D. (1998). Cómo mejorar la comprensión lectora. Madrid: Visor Fotocomposición. UPEL. Maracay. Universidad Pedagógica Experimental Libertador. Núcleo Biodiversidad.

De Zunino, D. L. (1985). La relatividad de la enseñanza y la relatividad de la comprensión: Un enfoque psicogenético. Lectura y vida, 6(4).

Echavarría, M. \& Gastón, I. (2000) Dificultades de comprensión lectora en estudiantes universitarios. Implicaciones en el diseño de programas de intervención. Revista de Psicodidáctica, Universidad del País Vasco, 010: 1-16.

Escalante, M. E. (2008). La comprensión lectora y el rendimiento académico entre los estudiantes del Pre-Grado de la Facultad de Educación de la UNMSM - 2008 - Perú. Universidad Nacional Mayor de San Marcos.

Flores, J. (2011). Hábitos lectores y competencias básicas en el alumnado de educación secundaria obligatoria. Educación XX1: Revista de la Facultad de Educación, ISSN 1139-613X, ISSN-e 2174-5374, 14 (1), 117-134

Giovannini, A. (1996), La comprensión lectora. Profesor en acción 3, Madrid, Edelsa

Hauy, M. E. (2014). Lectura literaria: aportes para una didáctica de la literatura. Zona Próxima, (20), 22-34. 
Volumen 2, Número 1, Enero-Abril 2021 Fecha de publicación: 15-03-2021 - Fecha de aceptación:14-02-2021

\section{GnosisWisdam}

Huamaní, L. B. (2008). Aplicación de estrategias metacognitivas para mejorar la comprensión lectora en alumnos de educación primaria. Perú: Puno. Universidad Nacional del Altiplano.

Huancas, A. (2019). Creatividad y producción literaria poética de estudiantes de literatura de la Universidad Nacional de Educación Enrique Guzmán y Valle. Universidad Nacional de Educación Enrique Guzmán y Valle.

Narváez, L. Á. (2016). Método de análisis para la comprensión de textos en estudiantes de la mención de lengua y literatura de la Universidad Nacional de Trujillo. Universidad Nacional de Trujillo.

Nina, K. (2018). Relación entre la comprensión lectora y el rendimiento académico en los estudiantes universitarios de la Escuela Profesional de Ingeniería Pesquera de La Universidad Nacional de San Agustín 2018. Universidad San Pedro.

Núñez, K. C. (2016). Relación entre dominio semántico y comprensión lectora en estudiantes de la especialidad de Lengua y Literatura, Facultad de Educación de la Universidad Nacional Federico Villarreal, 2013. Universidad Nacional Federico Villareal.

Ochoa, S. \& Aragón, L. (2004)
Funcionamiento metacognitivo de estudiantes universitarios durante la lectura de artículos científicos. Recuperado el 28 de abril de 2013 de: http://redaly c.uaemex.mx/pdf/647/64760303.pdf.

Pérez, J., \& Merino, M. (2008). Definicion.de: Concepto de producción. Retrieved from http://definicion.de/produccion/)

Ramos, M. (2010). El problema de la lectoescritura en el Perú: Desde la crisis institucional al urgente respeto de la psicogénesis en el segundo y el tercer ciclo de la EBR. Pontificia Universidad Católica del Perú.

Ramos, M. (2011). El problema de comprensión y producción de textos en el Perú. 9.

Rodríguez, M. Á. (2014). Propuesta didáctica basada en las técnicas de Gianni Rodari y los juegos de Edward de Bono para la producción de textos literarios creativos. Revista de Comunicación de la SEECI, (33), 117131.

Rueda, L. A., \& Turriago, M. L. (2003). La producción literaria como un proceso para mejorar el pensamiento, Universidad de La Sabana. https://doi.org/10.16309/j.cnki.issn.10 07-1776.2003.03.004

Real, T., Gutierrez, F., \& Quiroz, G. (2004). "Estrategias para el aprendizaje del pág. 55 


\section{Gnasis Wisdam}

educando". Universidad Nacional de Educación Enrique Guzmán y Valle La Cantuta.

Solé, I. (1994). Estrategias de Lectura. Barcelona: Graó.

Schmitt. (1998). "Los efectos de una actividad de lectura dirigida elaborado sobre las habilidades de metacomprensión". Chicago.

Ugarriza, N. (2006). La comprensión lectora inferencial de textos especializados y el rendimiento académico de los estudiantes universitarios del primer ciclo. Persona, (9), 31-75.

Vallés Arándiga, A. (2005). Comprensión lectora $y$ procesos psicológicos. Liberabit, 11(11), 41-48.

Zorrilla, M. J. P. (2005). Evaluación de la comprensión lectora: dificultades y limitaciones. Revista de educación, 126(nd).

Zuazo, N. (2017). Influencia de las estrategias de la comprensión lectora en la producción de textos narrativos del área de comunicación de los alumnos y alumnas de $5^{\circ}$ grado de educación primaria de la I.E. María Victoria Rumiche Fiestas $N^{\circ} 14083$, Parachique, Sechura-Piura, 2017 (Tesis). Universidad Católica Los Ángeles, Chimbote.

\section{CORRESPONDENCIA:}

Jorge Rosales Gallegos

rosalesgallegos7@gmail.com 